ARTICLE

Received 9 Dec 2015 | Accepted 27 Apr 2016 | Published 17 Jun 2016

\title{
Direct imaging of topological edge states at a bilayer graphene domain wall
}

Long-Jing Yin ${ }^{1, \star}$, Hua Jiang ${ }^{2, \star}$, Jia-Bin Qiao ${ }^{1} \&$ Lin $\mathrm{He}^{1}$

The AB-BA domain wall in gapped graphene bilayers is a rare naked structure hosting topological electronic states. Although it has been extensively studied in theory, a direct imaging of its topological edge states is still missing. Here we image the topological edge states at the graphene bilayer domain wall by using scanning tunnelling microscope. The simultaneously obtained atomic-resolution images of the domain wall provide us unprecedented opportunities to measure the spatially varying edge states within it. The one-dimensional conducting channels are observed to be mainly located around the two edges of the domain wall, which is reproduced quite well by our theoretical calculations. Our experiment further demonstrates that the one-dimensional topological states are quite robust even in the presence of high magnetic fields. The result reported here may raise hopes of graphene-based electronics with ultra-low dissipation.

\footnotetext{
${ }^{1}$ Center for Advanced Quantum Studies, Department of Physics, Beijing Normal University, Beijing 100875, China. ${ }^{2}$ College of Physics, Optoelectronics and Energy, Soochow University, Suzhou 215006, China. * These authors contributed equally to this work. Correspondence and requests for materials should be addressed to L.H. (email: helin@bnu.edu.cn).
} 
ooking for systems where topological edge states persist in the absence of external magnetic fields boosts rapid developments in condensed matter physics in the past few years ${ }^{1-15}$. Gapped graphene bilayer with smooth domain walls is predicted to be one of the most promising candidates where charge carriers can travel long distances with ultra-low dissipation ${ }^{8-12}$. The domain wall separating two oppositely biased bilayer graphene is first proposed by Martin et al. ${ }^{8}$ to host one-dimensional (1D) topological states. Later, the domain wall between AB- and BA-stacked bilayer graphene under a uniform external field is demonstrated to be equivalent to the gate-polarity domain wall ${ }^{8}$ and it is believed to be a crystalline topological defect hosting symmetry-protected topological gapless mode because of a change in the Chern number ${ }^{12}$. Very recently, the existence of topologically protected 1D edge states have been demonstrated explicitly in the two types of domain walls through transport measurement ${ }^{13,14}$, opening up opportunities for exploring unique topological states in graphene bilayer.

The AB-BA domain wall in graphene bilayer, with electrons residing right at the surface, provides unprecedented opportunities to directly image the topologically protected $1 \mathrm{D}$ conducting channels. More importantly, such a crystalline topological line defect exists naturally in Bernal graphene bilayers grown by chemical vapour deposition ${ }^{16,17}$ and in exfoliated bilayer graphene (that is, prepared using adhesive tape) from graphite ${ }^{93}$. Here we report direct imaging of the topologically protected $1 \mathrm{D}$ conducting channels in the AB-BA domain wall in exfoliated graphene bilayer. The exfoliated bilayer and trilayer graphene flakes were deposited on the substrate (here the supporting substrate is graphite) during the process of mechanical exfoliation and, very importantly, these graphene sheets decouple from the graphite surface due to the presence of the stacking misorientation with the underlying substrates, as demonstrated in this study and in previous studies ${ }^{18-25}$.

\section{Results}

The structure of AB-BA stacking domain wall. To identify the AB-BA domain wall in decoupled bilayer graphene on graphite, we used both the scanning tunnelling microscopy (STM) images (Fig. 1) and the scanning tunnelling spectroscopy (STS) spectra (Fig. 2). First, the decoupled bilayer graphene on graphite exhibits a small period of moiré patterns (that is, with a large rotation angle with the substrate) in the STM measurements ${ }^{19,21,25}$ and its atomic-resolution STM image shows a triangular lattice because of the A/B atoms' asymmetry in the topmost Bernal bilayer (see Fig. 1c and Supplementary Fig. 1). The decoupled monolayer graphene also exhibits a small period of moiré patterns; however, its atomic-resolution STM images show a hexagonal lattice (see Supplementary Fig. 1). The high-field STS spectra provide further information about the stacking orders of the topmost few layers $^{22}$ : the decoupled Bernal bilayer shows Landau quantization of massive Dirac Fermions (Fig. 2 and Supplementary Fig. 2) 22,26 , whereas the decoupled monolayer exhibits Landau quantization of massless Dirac fermions (see Supplementary Fig. 1) ${ }^{18,19}$.

Once the decoupled bilayer graphene is identified, we used STM measurements to find 1D structures (see Fig. 1c as an example) in the bilayer region as a possible candidate for the AB-BA domain wall. The strong dependence of the $1 \mathrm{D}$ structure on the bias voltage (used for imaging), as shown in Supplementary Fig. 3, excludes the graphene nanoripple ${ }^{27}$ or nanowrinkle ${ }^{28}$ as the origin of the $1 \mathrm{D}$ structure. We attribute the $1 \mathrm{D}$ structure in Fig. $1 \mathrm{c}$ to the AB-BA domain wall in bilayer graphene. We can observe the $\mathrm{AB}-\mathrm{BA}$ domain walls in the STM measurement, owing to their relatively higher conductivity comparing with that of the adjacent gapped bilayer regions (see Fig. 2f). The ultra-low random potential fluctuations due to substrate imperfections allows us to obtain high-quality atomic-resolution STM images of the domain wall, as shown in Fig. 1c,e. We obtained a triangular lattice, as is the characteristic a

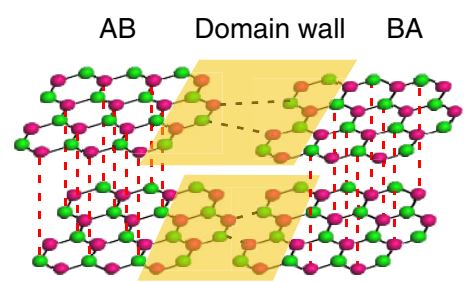

C

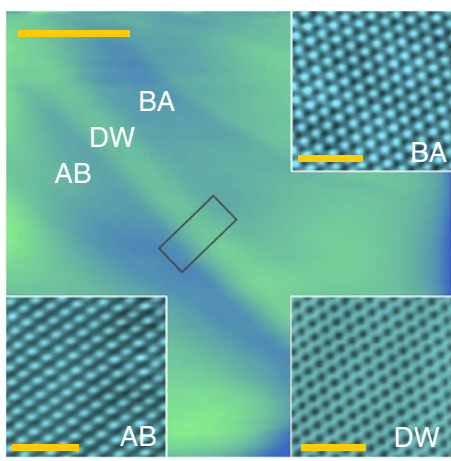

b

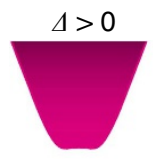

$\mathrm{AB}$
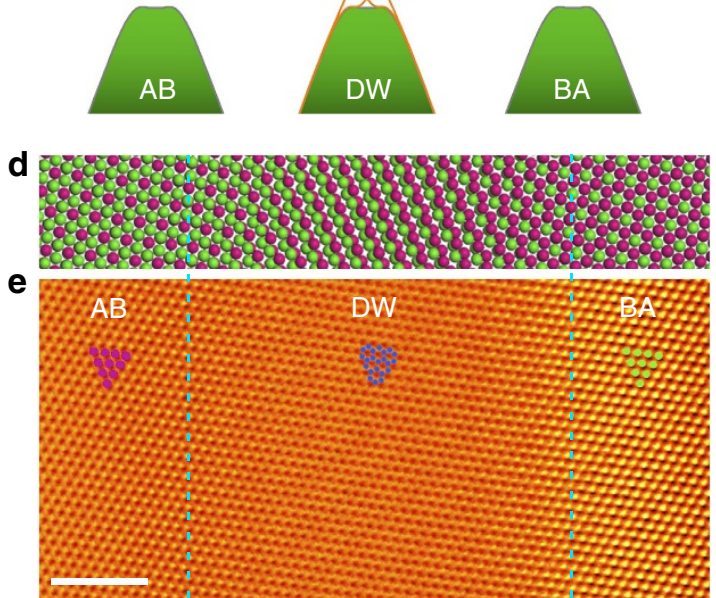

Figure 1 | AB-BA domain wall in bilayer graphene. (a) Schematic representation of an AB-BA domain wall in bilayer graphene. (b) Schematic band structures of the $A B$, domain wall (DW) and $B A$ regions of a bilayer graphene. The $A B$ - and $B A$-stacked regions are gapped. The topological edge states (orange curves) emerge in the DW region. (c) STM topographic image $(80 \times 80 \mathrm{~nm})$ of a decoupled bilayer graphene region on graphite surface $\left(V_{b}=0.4 \mathrm{~V}, I=0.25 \mathrm{nA}\right)$. An AB-BA domain wall is observed in the bilayer. Scale bar, 20 nm. Insets: atomic-resolution STM images in the AB, DW and BA regions, respectively. Scale bars, $1 \mathrm{~nm}$. (d) Schematic image of the $A B-B A$ domain wall. (e) $A$ typical atomic-resolution $S T M$ current image across the $A B-$ $\mathrm{BA}$ domain wall (rectangular region in c). A transition from triangular lattice (in the $A B$ region) to hexangular-like lattice (in the centre of the domain wall) and then to triangular lattice (in the BA region) is clearly observed. The width of the domain wall is estimated to be ( $8 \pm 1$ ) $\mathrm{nm}$. Scale bar, $2 \mathrm{~nm}$. 
a

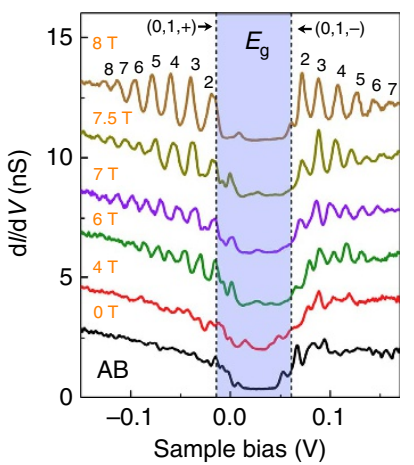

b

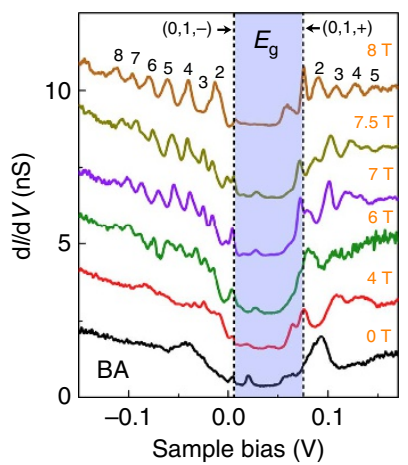

C

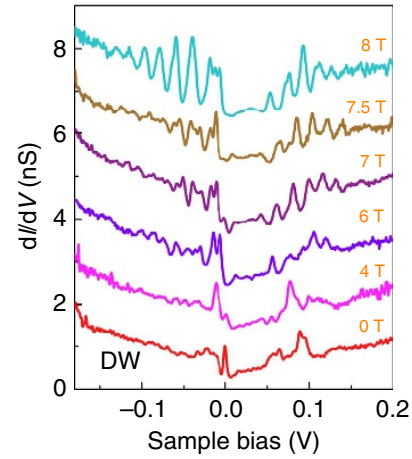

d
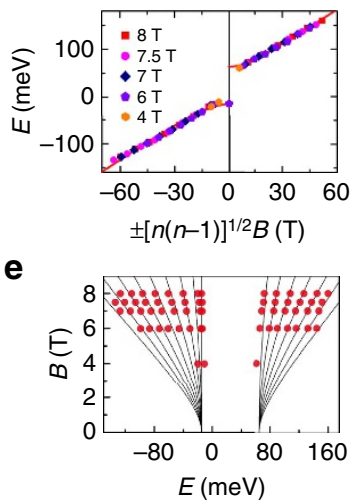

f

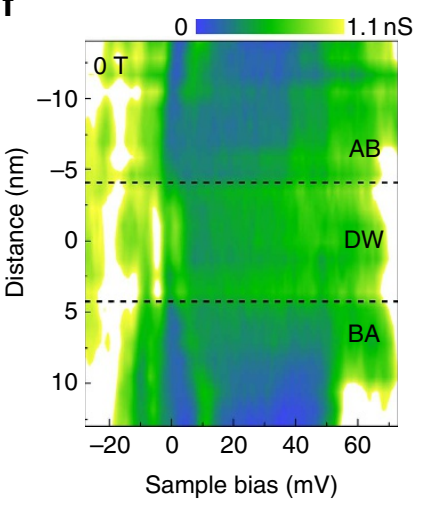

g

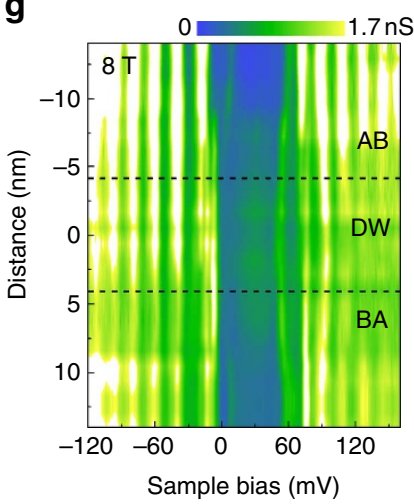

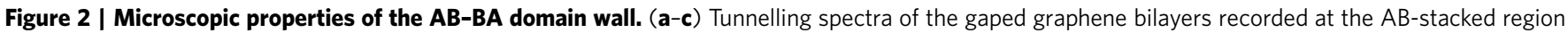
(a), the BA-stacked region (b) and the domain wall region (c) under various magnetic fields. LL peak indices are marked ( \pm are valley indices) and

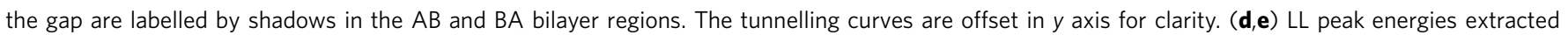
from a plotted versus $\pm[n(n-1)]^{1 / 2} B(\mathbf{d})$ and the magnetic fields $B(\mathbf{e})$. The solid curves are the fitting of the data with the theoretical equation

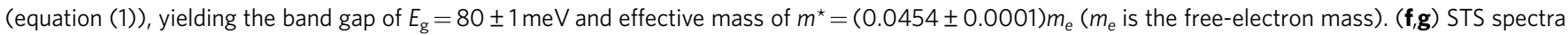
maps at $0 \mathrm{~T}(\mathbf{f})$ and $8 \mathrm{~T}(\mathbf{g})$ measured across the AB-BA domain wall. The zero position is defined at the middle of the domain wall.

of Bernal-stacked bilayer, both in the left and right regions, whereas we obtained a hexagonal-like lattice in the centre of the domain wall (see the insets of Fig. 1c). The 1D structure with hexagonal-like lattice at its centre, and $\mathrm{AB}$ and $\mathrm{BA}$ domains surrounding directly demonstrated that the studied structure is the $\mathrm{AB}-\mathrm{BA}$ domain wall. Figure $1 \mathrm{~d}$ shows a representative atomic-resolution STM image of the AB-BA domain wall. From left to right of the domain wall, the two graphene sheets translate relative to each other in opposite directions (one translates downward and the other translates upward), completing an interlayer translation from $\mathrm{AB}$ to $\mathrm{BA}$ stacking. The interatomic distances in the domain wall and in the Bernal bilayer regions were further analysed by taking a two-dimensional Fourier transform and the interatomic distances in the domain wall are $(1.5 \pm 0.5) \%$ smaller than those in the surrounding Bernal regions (Supplementary Fig. 4). To complete a one-bond-length armchair-direction interlayer translation from $\mathrm{AB}$ to $\mathrm{BA}$ stacking, the width of the domain wall is estimated to be $\sim 9.0 \mathrm{~nm}$ with the the measured lattice deformation $\sim 1.5 \%$, which agrees quite well with the measured value $\sim(8.0 \pm 1.0) \mathrm{nm}$. The angle between the boundary normal and the translation direction is measured to be about $85^{\circ}$, indicating that the studied domain wall is almost a purely shear soliton ${ }^{16}$.

Microscopic electronic properties of the $\mathrm{AB}-\mathrm{BA}$ domain wall. The electronic properties around the AB-BA domain wall are further studied by STS measurements, as shown in Fig. 2 and
Supplementary Figs 5 and 6 . The spectra recorded in both the $\mathrm{AB}$ - and BA-stacked regions exhibit characteristics that are expected to be observed in gapped graphene bilayers ${ }^{21,26,29}$. The substrate breaks the inverse symmetry of the topmost adjacent bilayers and then a finite gap $\sim 80 \mathrm{meV}$ is generated in the parabolic bands of the Bernal bilayer (the charge neutrality points of the two Bernal bilayer regions are measured to differ $\sim 15 \mathrm{meV})$. At the level of low-energy effective theory, the AB-stacked bilayer is equivalent to the BA-stacked bilayer subjecting to the opposite gate polarity ${ }^{12}$. Thus, the sign of the energy gap changes across the domain wall from the AB- to BA-stacked regions and symmetry-protected gapless modes are expected to emerge in the domain wall. In high magnetic fields, the spectra recorded in the Bernal bilayer regions exhibit Landau quantization of massive Dirac fermions (Fig. 2d,e and see Methods and Supplementary Information for further analysis). The two lowest Landau levels (LLs) $\mathrm{LL}_{(0,1,+)}$ and $\mathrm{LL}_{(0,1,-)}$ (here 0 / 1 are Landau indices and $+/-$ are valley indices), which are a couple of layer-polarized quartets, depends on the sign of the gate polarity (or the sign of the energy gap) of the two Bernal bilayer regions ${ }^{29}$. Therefore, they are reversed in the adjacent AB- and BA-stacked regions, as shown in Fig. 2a,b. In the experiment, the measured local density of states (LDOS) at position $r$ are determined by the wavefunctions, whereas the wavefunctions of LLs have their spatial extent, $\sim 2 \sqrt{N} l_{B}$ (here $N$ is the Landau index and $l_{B}=\sqrt{\hbar / e B}$ which is of the order of $10 \mathrm{~nm}$ for the magnetic fields applied in our experiment) ${ }^{26}$. Consequently, we can detect LLs of both the $\mathrm{AB}$ and $\mathrm{BA}$ domains in the spectra recorded in the 
AB-BA domain wall (Fig. $2 \mathrm{c}$ and see Supplementary Fig. 5 for details of calculation). The 'splitting' of the LLs recorded in the domain wall, as shown in Fig. 2c,g, arises from the relative shift of the charge neutrality points of the adjacent $\mathrm{AB}$ and $\mathrm{BA}$ domains. By using similar STM measurements, layer stacking domain walls in trilayer graphene, which separate ABA- and ABC-stacked trilayer graphene, have also been observed unambiguously in our experiment (see Supplementary Figs 7 and 8 for an example). Our result demonstrates that the layer-stacking domain walls naturally exist in graphene multilayers and affect their electronic properties dramatically.

Mapping of the topological states at the AB-BA domain wall. To further confirm the existence of symmetry-protected topological conducting channels in the $\mathrm{AB}-\mathrm{BA}$ domain wall, we directly imaged these $1 \mathrm{D}$ states by operating energy-fixed STS mapping, which reflects the LDOS in real space. Figure $3 \mathrm{a}-\mathrm{c}$ shows several STS maps at different energies. At the energies within the band gap of the adjacent $\mathrm{AB}$ and $\mathrm{BA}$ domains, clearly $1 \mathrm{D}$ conducting channels can be observed along the domain wall. A notable feature of the topological states is that they mainly located at the two edges of the $\mathrm{AB}-\mathrm{BA}$ domain wall and such a feature is independent of the energy of the gapless edge states. To verify the spatial distribution of the gapless states, we calculated electronic structures of a shear domain wall with a finite width $W$ (see Fig. 3d for an example). Figure 3e shows a schematic representation of the domain wall. In the calculation, we consider a tight-binding Hamiltonian with nearest-neighbour intra- and interlayer hopping and a finite chemical potential difference between two layers is taking into account to describe the energy gap observed in the Bernal regions ${ }^{12}$. The symmetry-protected gapless edge states emerge in the domain wall (Fig. 3d), which is irrespective of the type and width of the domain wall (see Methods and Supplementary Information for details of calculation). In our experiment, the STS maps probe predominantly the LDOS of the top layer. To compare with the experimental result, we plot a representative theoretical distribution of the gapless edge states in the topmost graphene layer in Fig. 3f. Obviously, the topological states are mainly located at the two edges of the domain wall and this feature is found to be independent of the probed energy within the gap of the Bernal bilayer regions. Here we should point out that such a spatial distribution of the topological states is independent of the edges of the domain wall (see Supplementary Fig. 9). Therefore, our experimental observations are reproduced quite well by the theoretical calculations. This provides direct and compelling evidence that the symmetry-protected topological edge states exist in the AB-BA domain walls of gapped bilayer graphene. In the experiment, the distributions of the edge states reveal notable asymmetry at various setting energies (Fig. 3a,b). This may result from the energy difference of the charge neutrality points between the adjacent $\mathrm{AB}$ and $\mathrm{BA}$ domains $(\sim 15 \mathrm{meV}$ in our sample) or the existence of the disorder potential near the domain wall (see Supplementary Fig. 10 for details of discussion).

\section{Discussion}

The STS maps of the gapless edge states are also measured in the presence of high magnetic fields (Fig. 3g). It is remarkable that these states are quite robust even in the highest magnetic field $\sim 8 \mathrm{~T}$ of our STM system. More importantly, the full width at half-maximum of topological states along the two edges of the domain wall decreases with increasing the magnetic fields (Supplementary Fig. 11), which may further diminish any possible scattering of the topological edge states along the $\mathrm{AB}-\mathrm{BA}$ domain walls. In a very recent transport measurement, it was also demonstrated that the topological feature of the gapless edge states is very robust against the perturbation of external magnetic fields and the backscattering of the topological states is further suppressed in the presence of magnetic fields ${ }^{14}$ Our work thus demonstrates the robust feature of the symmetry-protected topological edge states in the AB-BA domain walls of gapped bilayer graphene, opening a wide vista of graphene-based topological transport properties.
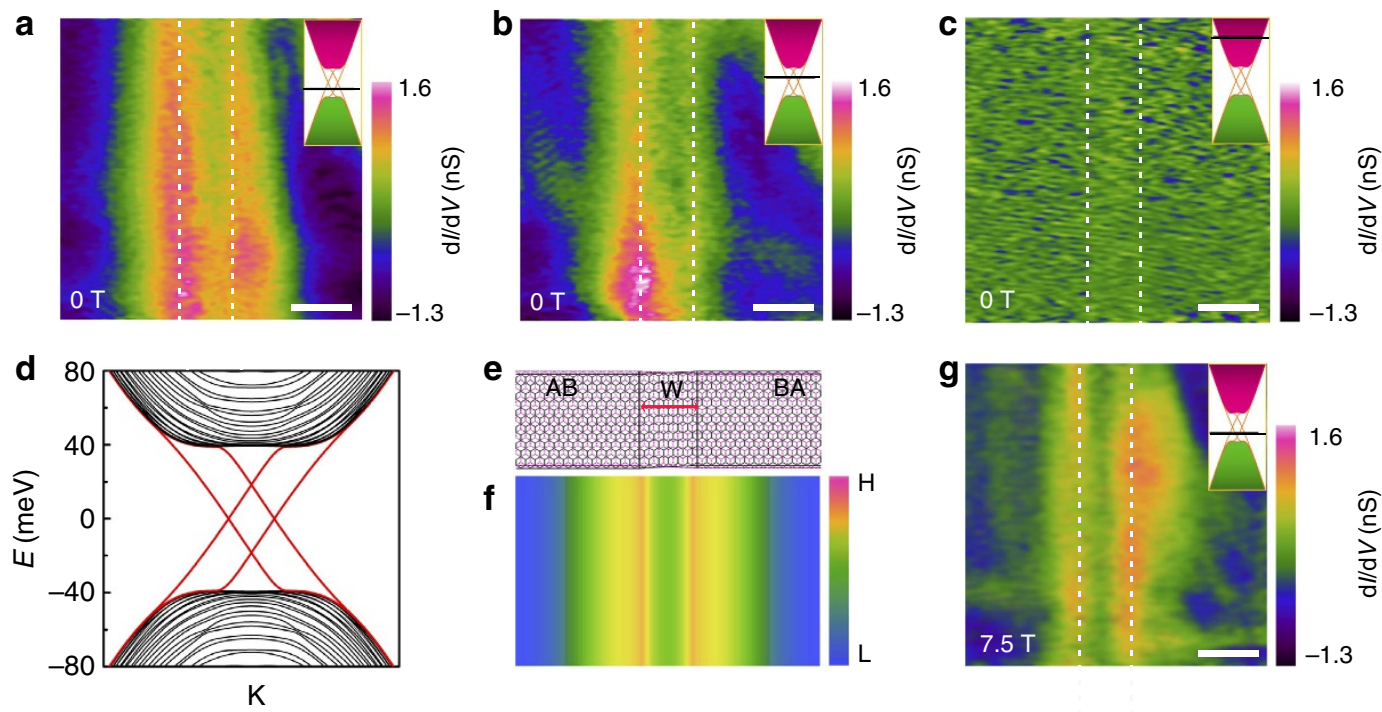

Figure 3 | Direct imaging of the 1D conducting channels at the AB-BA domain wall. (a-c) $d / / d V$ maps recorded under $O T$ along the $A B-B A$ domain wall with the fixed sample bias of $30 \mathrm{mV}(\mathbf{a}), 40 \mathrm{mV}(\mathbf{b})$ and $300 \mathrm{mV}(\mathbf{c})$, respectively. The 1D topological states are predominantly located at the two edges of the domain wall. (d) A representative theoretical band structure of an AB-BA domain wall with width of $8 \mathrm{~nm}$ and the gap in the Bernal region of $80 \mathrm{meV}$. (e) Illustration of an AB-BA domain wall. (f) Spatial distribution of the topological states around the domain wall obtained by theoretical calculation. (g) STS map of the domain wall taken at $7.5 \mathrm{~T}$ with sample bias of $30 \mathrm{mV}$. The two edges of the AB-BA domain wall are labelled by dashed lines. Scale bars, $10 \mathrm{~nm}$. 


\section{Methods}

Sample preparation and STM/STS measurements. The measurements were employed on highly oriented pyrolytic graphite surface. The highly oriented pyrolytic graphite samples were of ZYA grade (from NT-MDT) and were surface cleaved immediately by the adhesive tape method before experiments without any further processing. The STM system was an ultrahigh vacuum scanning probe microscope (USM-1500S) from UNISOKU with the magnetic fields up to $8 \mathrm{~T}$. All the STM and STS measurements were performed in the ultrahigh vacuum chamber $\left(\sim 10^{-11}\right.$ Torr $)$ with constant-current scanning mode. The experiments were acquired at temperature $\sim 4.5 \mathrm{~K}$. The STM tips were obtained by chemical etching from a wire of $\operatorname{Pt}(80 \%) \operatorname{Ir}(20 \%)$ alloys. Lateral dimensions observed in the STM images were calibrated using a standard graphene lattice and a Si (111)- $(7 \times 7)$ lattice and $\mathrm{Ag}(111)$ surface. The $\mathrm{d} I / \mathrm{d} V$ measurements were taken with a standard lock-in technique by turning off the feedback circuit and using a $793-\mathrm{Hz} 5 \mathrm{mV}$ a.c. modulation of the sample voltage. All the STS measurements were not recorded until the right standard tunnelling spectra of graphite was obtained.

Landau quantization in bilayer graphene. The LL sequences of gapped graphene bilayers can be described by ${ }^{29}$

$$
\begin{aligned}
& E_{n}=E_{C} \pm \sqrt{\left(\hbar \omega_{c}\right)^{2}[n(n-1)]+(U / 2)^{2}}-\xi z U / 4, \quad n=2,3,4 \ldots \\
& E_{0}=E_{C}+\xi U / 2, E_{1}=E_{C}+\xi(U / 2)(1-z)
\end{aligned}
$$

where $E_{\mathrm{C}}$ is the energy of charge neutrality point, $\omega_{c}=e \mathrm{eB} / \mathrm{m}^{*}$ is the cyclotron frequency, $m^{*}$ is the effective mass of charge carriers and $\xi= \pm$ are the valley indices. We have $z=2 \hbar \omega_{c} / t_{\perp}<<1$ for $B \leq 8 \mathrm{~T}$ and $|U| \approx E_{\mathrm{g}}$ (gap energy) when the interlayer bias $U<t_{\perp}$. The fitting results of experimental data to equation (1) are shown in Fig. 2 and Supplementary Fig. 2.

\section{Calculation of the topological edge states in the AB-BA domain walls.} To better understand the phenomena observed in the experiment, we further perform the numerical studies of the kink states in the AB-BA domain wall for comparison. We consider two typical AB-BA sandwich structures: (i) zigzag domain wall as illustrated in Supplementary Fig. 9d and (ii) armchair domain wall as illustrated in Supplementary Fig. $9 \mathrm{a}^{30}$. The domain wall in any geometry can be regarded as the mixture of these two typical domain wall structures. For both two structures, the domain wall region locates in $x \in\left[-\frac{W}{2}, \frac{W}{2}\right]$. The tight-binding Hamiltonian for AB-stacked bilayer Graphene region can be written as ${ }^{12}$ :

$$
\mathrm{H}=-t \sum_{l=1}^{2} \sum_{\langle i, j\rangle} a_{l, i}^{\dagger} b_{l, j}+\Delta \sum_{l=1}^{2} \sum_{i}(-1)^{l}\left(a_{l, i}^{\dagger} a_{l, i}+b_{l, i}^{\dagger} b_{l, i}\right)-t_{\perp} \sum_{i} a_{1, i}^{\dagger} b_{2, i}+H . C .
$$

where $a_{l, i}^{\dagger}\left(b_{l, i}^{\dagger}\right)$ is the creation operator for electron on site $i=(x, y)$ in layer $l$ on sublattice $A(B) ; l=1$ and 2 denote the top and bottom layer, respectively; $t$ and $t_{\perp}$ are the intralayer and interlayer hopping energy between nearest-neighbour sites; and $\Delta$ is the chemical potential between the top and bottom layer. These parameters are set as $t=2.8 \mathrm{eV}, t_{\perp}=0.4 \mathrm{eV}$ and $\Delta=0.06 \mathrm{eV}$. For BA-stacked region, the model is similar as equation (2), except that the interlayer term is replaced by $-t_{\perp} \sum b_{1, i}^{\dagger} a_{2, i}+H . c$. For the domain wall region, owing to the lattice misalignment, the interlayer hopping energy is much weaker than that in the $\mathrm{AB}(\mathrm{BA})$-stacked region. We also describe such region by equation (2) but set $t_{\perp}=0$. In the following numerical calculations, for the sake of the simplicity, both $\mathrm{AB}$ - and BA-stacked regions are modelled by the wide nanoribbon with open boundary condition. For the zigzag structure (see Supplementary Fig. 9), the whole system width is $264 \mathrm{~nm}$ and the domain wall width is $W=8.52 \mathrm{~nm}$. For the armchair structure (see Supplementary Fig. 9), the whole system width is $156 \mathrm{~nm}$ and the domain wall width is $W=8.40 \mathrm{~nm}$. First, we obtain the band structure for the domain wall structure. The studied system has the translational symmetry along the $y$ direction and the momentum $k$ in the $y$ direction is a good quantum number. Performing the partial Fourier transformation in $y$ direction, one obtain the Hamiltonian $H(k, l, x)$ under the bases of sites $[l, x]$. Here, $l$ denotes the layer index and $x$ denotes the position in $x$ direction. The energy band can be obtained by directly diagonalizing the $H(k, l, x)$. Next we solve the eigenmode of the system at fixed energy $E$. If $E$ collide with energy band at $k_{a}=k_{1}, k_{2}, \ldots k_{M}$, the system has $M$ eigenmode. The eigenstate of mode $k_{\mathrm{a}}$ can be obtained by solving the eigen equation $H\left(k_{\mathrm{a}}, l, x\right) \Psi_{\mathrm{l}, \mathrm{a}}(x)=E \Psi_{\mathrm{l}, \mathrm{a}}(x)$. The spatial distribution of the $k_{\mathrm{a}}$ mode is characterized by $\rho_{a}(l, x)=\left|\Psi_{l, a}(x)\right|^{2}$. If $\rho_{a}(l, x)$ mainly locates around the domain wall, $k_{\mathrm{a}}$ mode is the kink state. If $\rho_{\mathrm{a}}(l, x)$ is nearly uniform distributed in the whole sample, $k_{\mathrm{a}}$ mode is the bulk state. Finally, the spatial distribution for the carrier density of states in top layer at energy $E$ is calculated from

$\rho(E, x)=\sum_{a=1}^{M} \rho_{a}(l=1, x)$. In principle, $\rho(E, x)$ should be directly proportional to STM signal $\mathrm{d} I / \mathrm{d} V$ at position $x$ and energy $E$, which had been measured in our experiments.

The data that support the findings of this study are available from the corresponding author on request.

\section{References}

1. Kane, C. L. \& Mele, E. J. Quantum spin Hall effect in graphene. Phys. Rev. Lett. 95, 226801 (2005).

2. Kane, C. L. \& Mele, E. J. $Z_{2}$ topological order and the quantum spin Hall effect. Phys. Rev. Lett. 95, 146802 (2005).

3. Bernevig, B. A., Hughes, T. L. \& Zhang, S.-C. Quantum spin Hall effect and topological phase transition in HgTe quantum wells. Science 314, 1757-1761 (2006).

4. König, M. et al. Quantum spin Hall insulator state in $\mathrm{HgTe}$ quantum wells. Science 318, 766-770 (2007)

5. Yu, R. et al. Quantized anomalous Hall effect in magnetic topological insulators. Science 329, 61-64 (2010).

6. Chang, C.-Z. et al. Experimental observation of the quantum anomalous Hall effect in a magnetic topological insulator. Science 340, 167-170 (2013).

7. Du, L., Knez, I., Sullivan, G. \& Du, R. R. Robust helical edge transport in gated InAs/GaSb bilayers. Phys. Rev. Lett. 114, 096802 (2015).

8. Martin, I., Blanter, Y. \& Morpurgo, A. Topological confinement in bilayer graphene. Phys. Rev. Lett. 100, 036804 (2008).

9. Jung, J., Zhang, F., Qiao, Z. \& MacDonald, A. H. Valley-Hall kink and edge states in multilayer graphene. Phys. Rev. B 84, 075418 (2011).

10. Zhang, F., MacDonald, A. H. \& Mele, E. J. Valley Chern numbers and boundary modes in gapped bilayer graphene. Proc. Natl Acad. Sci. USA 110, 10546-10551 (2013).

11. Li, X., Zhang, F., Niu, Q. \& MacDonald, A. H. Spontaneous layer-pseudospin domain walls in bilayer graphene. Phys. Rev. Lett. 113, 116803 (2014).

12. Vaezi, A., Liang, Y., Ngai, D. H., Yang, L. \& Kim, E.-A. Topological edge states at a tilt boundary in gated multilayer graphene. Phys. Rev. X 3, 021018 (2013).

13. Ju, L. et al. Topological valley transport at bilayer graphene domain walls. Nature 520, 650-655 (2015).

14. Li, J. et al. Experimental observation of edge states at the line junction of two oppositely biased bilayer graphene. Preprint at arXiv:1509.03912 (2015)

15. Qiao, Z. et al. Current partition at topological channel intersections. Phys. Rev. Lett. 112, 206601 (2014).

16. Alden, J. S. et al. Strain solitons and topological defects in bilayer graphene. Proc. Natl Acad. Sci. USA 110, 11256-11260 (2013).

17. Butz, B. et al. Dislocations in bilayer graphene. Nature 505, 533-537 (2014).

18. Li, G., Luican, A. \& Andrei, E. Y. Scanning tunneling spectroscopy of graphene on graphite. Phys. Rev. Lett. 102, 176804 (2009).

19. Luican, A. et al. Single-layer behavior and its breakdown in twisted graphene layers. Phys. Rev. Lett. 106, 126802 (2011).

20. Song, Y. J. et al. High-resolution tunnelling spectroscopy of a graphene quartet. Nature 467, 185-189 (2010).

21. Miller, D. L. et al. Observing the quantization of zero mass carriers in graphene. Science 324, 924-927 (2009).

22. Yin, L.-J., Li, S.-Y., Qiao, J. -B., Nie, J.-C. \& He, L. Landau quantization in graphene monolayer, Bernal bilayer, and Bernal trilayer on graphite surface. Phys. Rev. B 91, 115405 (2015).

23. Xu, R. et al. Direct probing of the stacking order and electronic spectrum of rhombohedral trilayer graphene with scanning tunnelingmicroscopy. Phys. Rev. B 91, 035410 (2015).

24. Yin, L.-J., Qiao, J.-B., Zuo, W.-J., Li, W.-T. \& He, L. Experimental evidence for non-Abelian gauge potentials in twisted graphene bilayers. Phys. Rev. B 92, 081406 (R) (2015).

25. Yin, L.-J. et al. Landau quantization and Fermi velocity renormalization in twisted graphene bilayers. Phys. Rev. B 92, 201408 (R) (2015).

26. Yin, L.-J., Zhang, Y., Qiao, J.-B., Li, S.-Y. \& He, L. Experimental observation of surface states and Landau levels bending in bilayer graphene. Phys. Rev. B 93, 125422 (2016).

27. Bai, K.-K. et al. Creating one-dimensional nanoscale periodic ripples in a continuous mosaic graphene monolayer. Phys. Rev. Lett. 113, 086102 (2014).

28. Yan, W. et al. Strain and curvature induced evolution of electronic band structures in twisted graphene bilayer. Nat. Commun. 4, 2159 (2013).

29. Rutter, G. M. et al. Microscopic polarization in bilayer graphene. Nat. Phys. 7, 649-655 (2011).

30. Koshino, M. Electronic transmission through AB-BA domain boundary in bilayer graphene. Phys. Rev. B 88, 115409 (2013)

\section{Acknowledgements}

This work was supported by the National Basic Research Program of China (Grants Numbers 2014CB920903, 2013CBA01603 and 2014CB920901), the National Natural Science Foundation of China (Grant Numbers 11422430, 11374035 and 11374219), the programme for New Century Excellent Talents in University of the Ministry of Education of China (Grant Number NCET-13-0054), Beijing Higher Education Young Elite Teacher Project (Grant Number YETP0238). L.H. also acknowledges support from the National Program for Support of Top-notch Young Professionals. 


\section{Author contributions}

L.J.Y. performed the STM experiments and analysed the data. H.J. performed the theoretical calculations. L.H. conceived and provided advice on the experiment, analysis and theoretical calculation. L.H. and L.J.Y. wrote the paper. All authors participated in the data discussion.

\section{Additional information}

Supplementary Information accompanies this paper at http://www.nature.com/ naturecommunications

Competing financial interests: The authors declare no competing financial interests.
Reprints and permission information is available online at http://npg.nature.com/ reprintsandpermissions/

How to cite this article: Yin, L.-J. et al. Direct imaging of topological edge states at a bilayer graphene domain wall. Nat. Commun. 7:11760 doi: 10.1038/ncomms11760 (2016)

(c) (i) This work is licensed under a Creative Commons Attribution 4.0 International License. The images or other third party material in this article are included in the article's Creative Commons license, unless indicated otherwise in the credit line; if the material is not included under the Creative Commons license, users will need to obtain permission from the license holder to reproduce the material. To view a copy of this license, visit http://creativecommons.org/licenses/by/4.0/ 